\title{
STUDY TO ASSESS THE STATUS OF BUPIVACAINE HYDROCHLORIDE IN THE MANAGEMENT OF ESSENTIAL TRIGEMINAL NEURALGIA
}

\author{
SHOHDA KHATUN ${ }^{1}$, ASHIS KUMAR BISWAS ${ }^{2}$, RAJAN KARMAKAR ${ }^{2}$, SOJEEB DHAR ${ }^{2}$, MST. MAHBUBA KAFIA PARVIN ${ }^{3}$ \\ ${ }^{1}$ Senior Consultant, Department of Oral \& Maxillofacial Surgery, BSMMU, ${ }^{2}$ Department of Oral \& Maxillofacial Surgery, BSMMU, \\ ${ }^{3}$ Medical Officer, Department of Oral \& Maxillofacial Surgery, BSMMU
}

\begin{abstract}
Trigeminal neuralgia (TN) is a rare form of neuropathic facial pain characterized by severe paroxysmal pain in the face. The treatment of trigeminal neuropathic pain disorder is a major therapeutic challenge. Medical therapy often fails either due to poor responses to drugs or to unacceptable side effects and for those cases local anesthesia should be considered. Twenty patients (nine men and eleven women) who were diagnosed with TN previously and were not responsive to further medical treatment were selected for treatment. For this study, the affected nerve was blocked with $1.5 \mathrm{ml}$ of $0.5 \%$ bupivacaine $\mathrm{HCl}$. Patient's visual analogue scores (VAS) were recorded on preoperative day and on post operative at day 3, 7days, 15 days. There was a significant difference between mean preoperative and postoperative VAS value. Preoperative value was $83.10 \pm 6.06$, at postoperative 3 days was $39.60 \pm 7.86$, at postoperative 7 days was $16.25 \pm 6.46$ and at postoperative 15 days was $3.30 \pm 3.19$. So it can be concluded that administration of $1.5 \mathrm{ml}$ of $0.5 \%$ bupivacaine $\mathrm{HCl}$ nerve block at regular interval can be used as treatment for patients who are affected by the side effects from high-dose antiepileptic drugs.
\end{abstract}

Key words: Trigeminal neuralgia, Bupivacaine, Visual Analogue Score.

(Bangladesh J Physiol Pharmacol 2010; 26(1\&2) : 30-33

\section{INTRODUCTION}

Trigeminal neuralgia (TN) is a disease characterized by paroxysmal and refractory severe pain occurring along the trigeminal nerve. The pain is strictly limited to the distribution of the fifth cranial nerve and can involve one, two or even three branches. Bilateral cases are very rare ${ }^{1}$. The management of trigeminal neuralgia continues to be a major therapeutic challenge. Current treatments are mainly divided into medical treatments and surgery and medication is often the first-line treatment. Traditionally, patients are offered surgical options only when medications fail or severe side effects develop ${ }^{2}$. Although current treatment is initially medical, medical treatment fails in $30 \%$ of cases because of inadequate pain control or side effects of the drugs used ${ }^{3}$. Surgery can alleviate the pain, but is associated with morbidity and mortality and is not always effective ${ }^{4}$. Phenytoin, carbamazepine, clonazepam, gabapentin, and baclofen have also been used as anti neuralgic drugs ${ }^{5,6}$. Medical management with anticonvulsant (antiepileptic) drugs has debilitating side effects and the drugs eventually lose effectiveness ${ }^{7}$. The medical treatments (anticonvulsant medications) eliminate or significantly

Address of Correspondence: Dr. Shohda Khatun, Senior Consultant, Department of Oral \& Maxillofacial Surgery, BSMMU, Shahbag, Dhaka, Bangladesh, Email: shohdakhatun@yahoo.com reduce the pain in approximately $75 \%$ of patients and are considered the treatment of choice for incident cases of $\mathrm{TN}^{8}$. Unfortunately, the relief provided by medical therapy generally decreases over time. When medications fail to relieve TN pain attacks, it is important to reduce the risk of severe side effects of surgery and surgical sequelae seen in cranial surgery considering the mean age of TN patients. Nerve block with local anesthetics is appropriate in such cases because this treatment is reversible and non traumatic so it can be effectively used. In this study we used nerve block with a long-lasting local anesthetic for treating TN as a minor intervention to decrease the risk of intracranial neurosurgery complications, eliminate the disadvantages of other peripheral interventions and patient suffering from side effects of carbamazipine or on whom carbamazipine is no longer effective as a treatment of trigeminal neuralgia and presenting a solution for patients in such difficult situation.

\section{MATERIALS AND METHODS}

The study involved 20 patients (nine men and eleven women) with essential trigeminal neuralgia. The study protocol was explained to the patients in detail before obtaining the informed consent from the patients. Only patients suffering from paroxysmal pain were included in order to standardize and focus the study. The diagnosis 
of idiopathic TN was based on paroxysmal attacks of pain in one or several branches of the trigeminal nerve with pain-free intervals trigger areas, pain-triggering stimuli and absence of signs of a trigeminal nerve organic lesion. All the patients were suffering from various side effect of carbamazipine as altered liver function and some of the patients had attack of Steven Jonson syndrome were included for study. The patient who had history of any previous surgical treatment by cryo-surgeryor microvascular decompression and alcohol block were excluded.

\section{Evaluation}

Visual an alogue scale (VAS; 100- $\mathrm{mm}$ line with 0 signifying no pain and $100 \mathrm{~mm}$ signifying worst pain imaginable) was used to measure pain. Torough discussion was done with the patient before procedure about the visual analogue scale ${ }^{9}$. Patient were instructed to score pain pre-operatively. Then $1.5 \mathrm{ml}$ of $0.5 \%$ bupivacanine injection is given at each branch of affected nerve. Then carefully scored the pain by patient at 3 days. If patient complains of moderate pain then another increased dose of bupivacaine $(3 \mathrm{ml}$ ) was given and scored after 7 days. If further patient complained of pain then another increased dose of (maximum $10 \mathrm{ml}$ ) bupivacaine is given at specific branch and observed for 15 days. Then patient was again instructed to score the pain.

\section{Data collection technique and data analysis}

After the completion of the procedure, a standardized structured data collection sheet was used to collect necessary information of the study subject. Patients were stratified by gender and affected branch.Clinical characteristics of the samples, and pre nerve block and post nerve block VAS scores were evaluated by the twosample paired t test. The data were analyzed using SPSS 10.0 .

\section{RESULT}

The mean age of the patients was $50.55 \pm 8.13$ years (Table 1). In our study group, 8 patients had been suffered from maxillary and 12 from mandibular trigeminal neuralgia (Table 1). They were taking carbamazepine for $5.35 \pm 1.26$ years. There was a significant difference between mean preoperative and postoperative VAS value at day 3, 7days, and 15 days. Preoperative score was $83.10 \pm 6.06$, at postoperative 3 days score was $39.60 \pm$ 7.86 , at postoperative 7 days was $16.25 \pm 6.46$ and at postoperative 15 days was $3.30 \pm 3.19$. From paired sample test (T test) it was found that the VAS score was highly significant when comparison was done between preoperative scores with postoperative 3 days, 7days, 15 days individually.
Table-I

Demographic profile of the patients at the beginning of the study

Mean \pm SD

Age

$50.55 \pm 8.13$

Time of disease treated with

$5.35 \pm 1.26$

carbamazipine

Male:Female ratio

Maxillary nerve involvment

Mandibular nerve involvment 12

Table-II

Mean VAS with Std at preoperative, postoperative 3 days, 7days, 15 days and comparison of preoperative mean VAS with postoperative 3 days, 7days, 15 days mean VAS

\begin{tabular}{rc}
\hline VAS Score & $P$ \\
Mean $\pm S D$ & value \\
\hline
\end{tabular}

pre-operative

$83.10 \pm 6.06$

Response of 1 st dose at 3 days $39.60 \pm 7.860 .000$ *

Response of 2 nd dose at 7 days $16.25 \pm 6.460 .000 *$

Response of 3rd dose at 15 days $3.30 \pm 3.19 \quad 0.000^{*}$

$P$ value $<0.05$ is significant. * means highly significant.

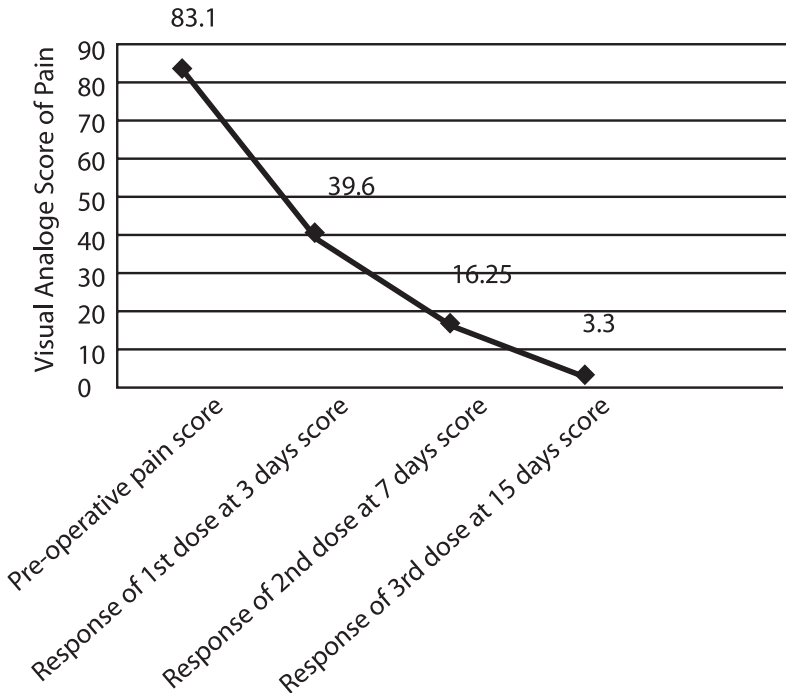

Fig.-1: Change of patient VSA means after bupivacaine $\mathrm{HCL}$ administration at affected nerve branch

\section{DISCUSSION}

The ideal goal of the management of trigeminal neuralgia is to achieve long-term total analgesia. Goto et $\mathrm{al}^{10}$ and Radwan et al ${ }^{11}$ found peripheral nerve block 
using high concentrations of local anesthetics prolongs the analgesic effect in patients with TN, without adverse effects. Umino et $\mathrm{al}^{12}$ reported that TN block with local anesthetics is reversible and non traumatic and is appropriate for further surgical interventions such as microvascular decompression. Amir et $\mathrm{al}^{13}$ Clinical and experimental data indicate that changes in the expression of voltage-gated sodium channels play a key role in the pathogenesis of neuropathic pain and that drugs that block these channels are potentially therapeutic in TN. In addition, recent data show that local anesthetics may have pain-relieving actions at targets other than sodium channels; these targets include neuronaln G proteincoupled receptors and binding sites on immune cells. One postulated mechanism for the long-term effect of local anesthetics on the trigeminal nerve is Wallerian degeneration. Histologically, the extrafascicular administration of local anesthetics at clinical concentrations can alter perineural permeability, causing endoneurial oedema, increased endoneurial fluid pressure, and Wallerian degeneration with Schwann cell injury and axonal dystrophy ${ }^{14}$ which may reduce allodynia, hyperalgesia, and trigger point hypersensitivity. Seventeen patients with long-lasting idiopathic trigeminal neuralgia were treated by Stajcic et $\mathrm{al}^{15}$. with weekly peripheral streptomycin/lidocaine (S/L) or lidocaine alone injections, in a double blind controlled study. Eight patients responded initially to the treatment in the $S / L$ group and three patients in the lidocaine group. Pain recurred in four patients from the $S / L$ group within two weeks and six months following the last injection. One patient from the lidocaine group remained pain-free for eight months. At the final assessment, three patients from the $S / L$ group and two patients from the lidocaine group remained pain-free up to 30 months. They also reported that sensory functions of the injected nerves were not affected after repeated injections as in our study. Continuous nerve block with bupivacaine $\mathrm{HCl}$ using an indwelling catheter has not been studied for oro-facial pain control, although this catheter technique has been used frequently and successfully in the epidural space to safely manage limb, chest, and abdominal pain ${ }^{16}$. There has been only one previously published case report describing a long-term continuous trigeminal nerve block with local anesthetics using a pain pump and a temporary indwelling catheter ${ }^{12}$. In that case, patientcontrolled analgesia via a pump injection was used with no side effects during local anesthetic infusion, as in our study. In a few case reports on the clinical application of high-concentration local anesthetics for the treatment of $\mathrm{TN}$, pain relief lasted for 2.2 weeks to 14 months ${ }^{10,11,13}$. Goto et al. ${ }^{10}$ used an infraorbital nerve block with $4 \%$ tetracaine dissolved in $0.5 \%$ bupivacaine to treat older TN patients who did not wish to have a neurolytic block or surgical treatment, and reported that the analgesic effects continued for more than 3 months. Sato et al. reported two cases of idiopathic superior laryngeal neuralgia treated with a superior laryngeal nerve block using a high concentration of lidocaine; the pain was alleviated for 1 year without the need to continue block therapy after 10 treatments using $1 \mathrm{~mL}$ of $10 \%$ lidocaine over 12 days. They postulated that the effective period in previous cases was shorter because the injected local anesthetic remained in the trigger zone for a shorter time ${ }^{17}$. Change of patient VAS means $0.5 \%$ bupivacaine $\mathrm{HCL}$ administration at specific nerve branch, goal of managing TN is to achieve long-term total analgesia, while preserving the sensory functions of the trigeminal nerve. We were able to provide pain relief for without jeopardizing sensory function. In addition, this method allows patients to continue their daily social activities without antiepileptic drugs, which can cause a lack of concentration, drowsiness, and dizziness.

\section{CONCLUSION}

The nerve block with $1.5 \mathrm{ml}$ of $0.5 \%$ bupivacaine injection for those patients who are unable to tolerate high doses of antiepileptic drugs, we observed a significant improvement in pain relief with this method without surgery.

\section{REFERENCES}

1. Umino M, Kohase H, Ideguchi S, Sakurai N. Long term pain control in trigeminal neuralgia. Clin J Pain 2000; 18:196199.

2. Nurmikko TJ, Eldridge PR. Trigeminal neuralgia pathophysiology, diagnosis and current treatment. $\mathrm{Br} \mathrm{J}$ Anaesth 2001; 87:117-173.

3. Sillanpaa M. Carbamazepine pharmacology and clinical use. Acta Neurol Scand 1981; 88:115-9.

4. Patsalos PN. Medical management. In Zakrzewska JM, ed. Trigeminal neuralgia: a practical approach to management, major problems in neurology series, vol 28. London: WB Saunders 1995: 80-107.

5. Zakrzewska JM, Patsalos PN. Drugs used in the management of trigeminal neuralgia. Oral Surg Oral Med Oral Pathol Oral Radiol Endod 1992; 74:439-50.

6. Umino M, Ohwatari $\mathrm{T}$, Shimoyama $\mathrm{K}$, et al. Long-term observation of the relation between pain intensity and serum carbamazepine concentration in elderly patients with trigeminal neuralgia. J Oral Maxillofac Surg 1993; 51:1338-44.

7. Taylor JC. Long-term treatment of trigeminal neuralgia with carbamazepine. Postgrad Med J 1981; 57:16-18.

8. Fields HL. Treatment of trigeminal neuralgia. N Engl J Med 1996; 334:1125-1126. 
9. Khatun S, Mollah MR, Akhtaruzzaman AKM, Rahman MS. Effectiveness of Multimodal Preemptive Analgesic Therapy in Maxillofacial Surgery. Bang J Physiol Pharmacol 2008; 24: 17-24.

10. Goto F, Ishizaki K, Yoshikawa D, Obata H, Arii H, Terada M. The long lasting effects of peripheral nerve blocks for trigeminal neuralgia using a high concentration of tetracaine dissolved in bupivacaine. Clin J Pain 1999; 79:101-103.

11. Radwan IAM, Saito S, Goto F. High-concentration tetracaine for the management of trigeminal neuralgia: quantitative assessment of sensory function after peripheral nerve block. Clin J Pain 2001; 17:323-326.

12. Umino $M$, Kohase $H$, Ideguchi $S$, Sakurai N. Long-term pain control in trigeminal neuralgia with local anesthetics using an indwelling catheter in the mandibular nerve. Clin J Pain 2002; 18:196-199.

13. Amir R, Argoff CE, Bennett GJ, Cummins TR, Durieux ME, Gerner $\mathrm{P}$, et al. The role of sodium channels in chronic inflammatory and neuropathic pain. Clin J Pain 2006; 7:129.

14. Myers RR, KalichmanMW, Reisner LS, Powell HC. Neurotoxicity of local anesthetics: altered perineurial permeability, edema, and nerve fiber injury. Anesthesiology J 1986; 64: 29-35.

15. Stajcic Z, Juniper RP, Todorovic U. Peripheral streptomycin/ lidocaine injections versus lidocaine alone in the treatment of idiopathic trigeminal neuralgia. A double blind controlled trial. J Craniomaxillofac Surg 1990; 18: 243-246.

16. Symreng T, Gomez MN, Johnson B, Rossi NP, Chiang CK. Intrapleural bupivacaine: technical considerations and intraoperative use. J Cardiothorac Anesth 1989; 3 : $139 \mathrm{e} 141$.

17. Sato TK, Suzuki M, Izuha A, Hayashi S, Isosu T, Murakawa M. Two cases of idiopathic superior laryngeal neuralgia treated by superior laryngeal nerve block with a high concentration of lidocaine. J Clin Anesth 2007; 19: 237238. 